\title{
UNTERSUCHUNGEN ZUR DEUTSCHEN SCHREIBSPRACHE EINES SPÄTMITTELALTERLICHEN GEBETBUCHES VON LJUBLJANA
}

\section{Vorbemerkung}

Der vorliegende Aufsatz ${ }^{1}$ bietet eine ausführliche systematische Darstellung der deutschen Schreibsprache des Manuskriptes NUKLj Ms 224, eines spätmittelalterlichen Gebetbuches der National- und Universitätsbibliothek in Ljubljana, welches drei verschiedene Hände aufweist. ${ }^{2}$ Die Schreibsprache wird nach den für räumliche und zeitliche Einordnung relevanten Kriterien in drei Bereichen untersucht: im phonetisch-orthographischen, im morphologischen und im lexikalischen. Den methodologischen Untersuchungsansatz bildet ein Vergleich zwischen dem "Normalmhd." (H. Paul 1989, 19 u. 28ff.) und dem in der Handschrift in Erscheinung tretenden Sprachzustand unter Berücksichtigung von J. Stanoniks (1957, 41) Bestimmung der Schreibsprache als Südbairisch und der mit Hilfe des Wasserzeichens bestimmbaren Datierung der Handschrift um 1472.3 Aufgrund

1 Er ist Teil meiner Magisterarbeit, die ich am 24.11. 1995 an der Philosophischen Fakultät der Universität Ljubljana verteidigt habe. Mitglieder der Prüfungskommission waren Prof. Dr. Anton Janko, Prof. Dr. Janez Stanonik und Prof. Dr. Neva Šlibar.

2 In den bisherigen kodikologischen Beschreibungen (M. Kos u. F. Stele 1931, 116; J. Stanonik 1957, 41) wird dagegen Hand 3 nicht eigens aufgeführt, doch deutet die als oberdeutsche Bastarda des 15. Jhs. bestimmte Schrift in ihrem Duktus klare Unterschiede zu Hand 2 auf. So hat das $h$ von Hand 3 im Gegensatz zu dem von Hand 2 auch eine Schleife in der Unterlänge, außerdem hat das $g$ bei Hand 3 eine Schleife, die mit dem nächsten Buchstaben verbunden ist, während das $g$ des zweiten Schreibers durch einen offenen Bogen gekennzeichnet ist; ferner treten Abweichungen in der Großschreibung auf, um nur einige Beispiele zu nennen. Wenn der letzte auf fol. $80^{v}$ geschriebene Text nicht von einem anderen Schreiber aufgezeichnet worden sein sollte, so hat die Schrift des zweiten unleugbare Veränderungen erfahren, die vielleicht auf eine spätere Eintragung hindeuten könnten.

3 In Anbetracht dessen, daß das Wasserzeichen auf einem Blatt nicht vollständig vorhanden ist und anhand von zwei aufeinanderfolgenden Blättern derselben Lage zu einem ganzen komplementiert werden muß, also sicher als zusammengehörig betrachtet werden kann, erweist sich die Wasserzeichenbestimmung nicht als unproblematisch, zumal eine exakte Pauszeichnung aus technischen Gründen nicht angefertigt werden konnte. Die Teile des Wasserzeichens sind zur Bogenmitte hin verschoben. Den Bindedrähten und anderen Einzelheiten nach zu urteilen, handelt es sich höchstwahrscheinlich um das Papierzeichen Waage Nr. V 563 nach der Wasserzeichenkartei 
fehlender textimmanenter Angaben zur exakten Datierung soll die Untersuchung der Schreibsprache dazu beitragen, die Entstehungszeit der Aufzeichnung näher zu bestimmen.

Als Wegweiser bei der sprachlichen Analyse wurden folgende Werke herangezogen: W. Besch 1967, O. Mausser 1933, $11 \mathrm{ff}$. sowie H. Paul 1989, 167ff. und 567f. Sehr aufschlußreich bei der Behandlung der Einzelprobleme waren neben der oben angeführten Literatur insbesondere V. Mosers Frühneuhochdeutsche Grammatik (1929 und 1951), die 1993 veröffentlichte, von der Autorengemeinschaft R. P. Ebert, O. Reichmann, H.-J. Solms und K.-P. Wegera verfaßte Frühneuhochdeutsche Grammatik ${ }^{4}$ und $K$. Weinholds, in den einschlägigen Standardwerken noch immer vielfach zitierte Bairische Grammatik aus dem Jahre 1867.

Wegen zum Teil bestehender Abweichungen in den eingangs erwähnten Bereichen muß bei der Untersuchung jeder Schreiber für sich berücksichtigt werden.

\section{Beobachtungen zur Lautung und Schreibung}

\subsection{Zum Vokalismus}

2.1.1 Zum Vokalismus der haupttontragenden Silben

2.1.1.1 Durchführungen der nhd. Diphtongierung 5

\subsection{Mhd. $\hat{\imath}>e i$}

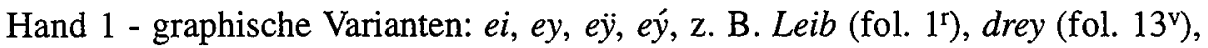
$e \ddot{y}$ (en (fol. $44^{\mathrm{v}}$ ), beý (fol. $\left.22^{\mathrm{v}}\right) .^{6}$

Hand 2 - graphische Varianten: ei, ey, z. B. itweicz (fol. 51 ${ }^{\mathrm{r}}$ ), dreyn (fol. $\left.65^{\mathrm{r}}\right){ }^{7}$

Hand 3 - nur $e i$, z. B. reich (fol. $\left.80^{\mathrm{v}}\right) .^{8}$

\subsection{Mhd. $\hat{u}>$ au}

Hand 1 - graphische Varianten: $a u, a u ́, a w$, z. B. taufent (fol. $7^{\mathrm{v}}$ ), daúmellen (fol. $48^{\mathrm{r}}$ ), haws (fol. 34v).

von G. Piccard (1978, 201), das für 1472 in Gemona del Friuli nachgewiesen ist.

4 Im folgenden abgekürzt als FrnhdG.

5 Im Bair., von den alem. Mundarten nur im Schwäbischen, vgl. W. Besch 1967, 75f. Siehe auch V. Moser 1929, 154ff.; FrnhdG 1993, $64 \mathrm{ff}$.

6 Aber: driualtigkait (fol. $4^{\mathrm{r}}, 10^{\mathrm{r}}, 13^{\mathrm{r}}, 14^{\mathrm{r}}, 27^{\mathrm{r}}$ ), driualtigkaitt (fol. 14 $\mathrm{r}, 29^{\mathrm{v}}, 47^{\mathrm{r}}$ ), drivaltigkaitt (fol. $42^{v}$ ), driualltigkait (fol. 49v). Das Unterbleiben der Diphtongierung ist hier lexemgebunden, es beschränkt sich nur auf die angeführten Beispiele; vgl. auch W. Besch 1967, 75.

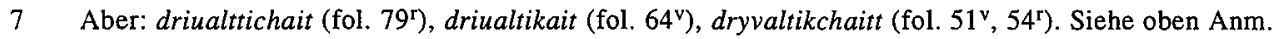
6.

8 Das Faktitiv Swigen (< mhd. sweigen, vgl. M. Lexer 1986, 221) deutet nicht auf fehlende Diphtongierung, sondern eher auf Nachlässigkeit des Schreibers. 
Hand 2 - nur au, z. B. auffart (fol. 79r).

Hand 3 - kein Beispiel.

2.1.1.1.3 Mhd. $i u>e u$

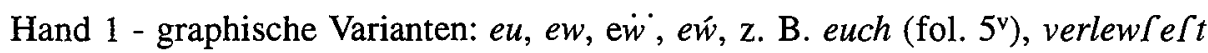
(fol. $1^{\mathrm{r}}$ ), gepew $\ddot{t}$ (fol. 50r), trew (fol. 41 ${ }^{\mathrm{r}}$ ).

Hand 2 - graphische Varianten: eu, ew, ev, ${ }^{9} \mathrm{z}$. B. freuntt (fol. $78^{\mathrm{v}}$ ), rew (fol. $67^{\mathrm{v}}$ ), trev (fol. 68 $8^{\mathrm{r}}$ ).

Hand 3 - nur $e u$ in euch (fol 80v).

2.1.1.2 Mhd. ou $>a u^{10}$

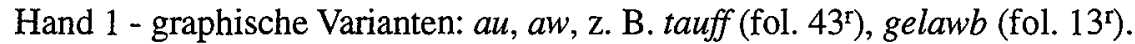

Hand 2 - graphische Varianten: $a u, a v,{ }^{11} a w$, z. B. augen (fol. 51 ${ }^{\mathrm{r}}$ ), fraven (fol. 51 ${ }^{\mathrm{r}}$ ), frawn $\left(51^{v}\right)$.

Hand 3 - nur au in haupt (fol. 80v).

2.1.1.3 Vereinzelte Durchführung der "jüngeren" Diphtongierung von $i>i e$ vor $r, h$ oder Nasal ${ }^{12}$

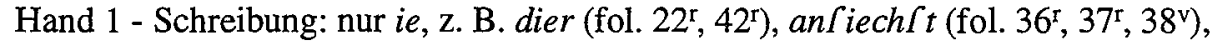
Siech (fol. $38^{\mathrm{r}}$ ). ${ }^{13}$

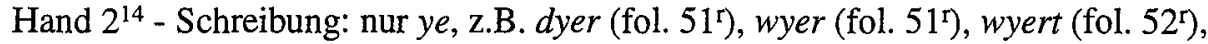
hyeml (fol. $\left.57^{\mathrm{r}}\right) .{ }^{15}$

Hand 3 - kein Beispiel.

\subsubsection{Unterbleiben der md. Monophtongierung ${ }^{16}$}

2.1.1.4.1 Erhaltung des mhd. ie

Hand 1 - graphische Varianten: ie, ye, ye, ýe, ${ }^{17}$ z. B. Spiegel (fol. $2^{\mathrm{r}}$ ), Nyemant (fol. $10^{\mathrm{r}}$ ), dÿennerin (fol. $\left.16^{\mathrm{v}}\right)$, ýemandt ${ }^{18}$ (fol. $18^{\mathrm{v}}$ ).

9 Die letzte Variante ist selten. Nach V. Moser $(1929,33)$ ist die Schreibung mit $v$ als zweitem Bestandteil schon im 15. Jh, verschwunden. In der FrnhdG wird sie dagegen als eine der Nebenformen angegeben, die "bes. in Hss. des 14./15. Jhs." vorkommen $(1993,61)$.

10 Vgl. W. Besch 1967, 81 u. 83.

11 Vgl. oben Anm. 9. - In frav und dessen Komposita fast ausschließlich av.

12 Besonders bair.-ostfrk., vgl. H. Paul 1989, 169f. - "Im Laufe des 15. Jhs. wird diese Veränderung, die übrigens im Südbairischen nie recht heimisch gewesen sein dürfte, im Bairischen wieder rückgängig gemacht (...)" Kranzmeyer 1956, 41f. Siehe auch V. Moser 1929, $130 \mathrm{f}$.

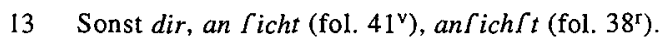

14 Häufiger als bei Hand 1.

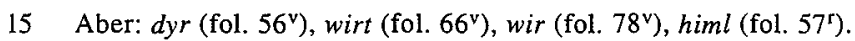

16 Vgl. V. Moser 1929, 190ff.; FrnhdG 1993, 62 f.

17 Die beiden letzten Graphien sind selten.

18 Wahrscheinlich diphtongischer Anlaut im Obd. (FrnhdG 1993, 68). 
Hand 2 - graphische Varianten: ie, ye, $\ddot{y e},{ }^{19} \mathrm{z}$. B. liebn (fol. $51^{\mathrm{v}}$ ), dyen $\mathrm{t}$ (fol. $78^{\mathrm{v}}$ ),

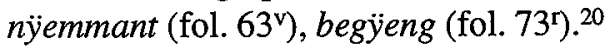

Hand 3 - kein Beispiel.

2.1.1.4.2 mhd. $u o>$ mittel- u. südbair. $u e^{21}$

Hand 1 - graphische Varianten: ue, úe, ${ }^{22}$ z. B. brueder (fol. $2^{\mathrm{v}}$ ), fúechet (fol. $38^{\mathrm{r}}$ ).

Hand 2 - nur ue, z. B. tuen (fol. $75^{\mathrm{v}}$ ). ${ }^{23}$

Hand 3 - kein Beispiel.

\subsection{Erhaltung des mhd. $\ddot{u} e^{24}$}

Hand 1 - graphische Varianten: üe, úe, ue, z. B. müe $\Upsilon e$ (fol. $11^{v}$ ), múe $\int e$ (fol. $32^{\mathrm{r}}$ ), mue ffe (fol. $2^{\mathrm{v}}$ ).

Hand 2 - ue, z. B. betruebten (fol. 68 $){ }^{25}$

Hand 3 - als $u^{26}$ in gru厂 (fol. $80^{v}$ ).

2.1.1.5 Mhd. $e i>a i^{27}$

Hand 1 - graphische Varianten: ai, ay, aÿ, aý, z. B. ain,$^{28}$ (fol. $1^{\mathrm{v}}$ ), gai $t^{29}$ (fol. $7^{\mathrm{r}}$ ), prait (fol. $1^{\mathrm{r}}$ ), raynunge (fol. $9^{\mathrm{v}}$ ), I waÿr trophen (fol. $2^{\mathrm{r}}$ ), haýll ${ }^{30}$ (fol. $14^{\mathrm{v}}$ ).

Hand 2 - graphische Varianten: ai, ay, z. B. $a i^{31}$ (fol. 78v), czwainczig (fol. 78 ), czwayn (fol. 65r).

19 Nur bei den angeführten Beispielen.

20 Vereinzelt kommen auch Monophtonge vor, z. B. vyrczig (fol. 79v).

21 Im 13. Jh., siehe H. Paul 1989, 170.

22 Aber: $u, u$, z. B. in guts (fol. 10 $0^{\mathrm{r}}$, mút (fol. $8^{\mathrm{r}}$ ). Das einfache Zeichen für den Diphtong setzt sich allmählich in der Schriftsprache durch, entspricht aber nicht dem tatsächlichen Lautwert. In bair. Hss. des 14. und des 15. Jhs. betrachtet V. Moser das Fehlen der Diphtongbezeichnung als "Schreibernachlässigkeit" (1929, 190); zur Durchsetzung der Monophtonge in der Schriftsprache siehe auch FrnhdG 1993, 62. - Die digraphische Schreibung ist hier vorherrschend.

23 Aber: $u$ (vgl. oben Anm. 22), das beim zweiten Schreiber überwiegt, z. B. tun (fol. $73^{r}$ ).

24 Zur Problematik des Umlauts (das Nebeneinander von Umlautung vs. Nichtumlautung einerseits und Bezeichnung vs. Nichtbezeichnung des Umlauts andererseits) siehe FrnhdG 1993, 34f.; vgl. auch V. Moser 1929, 26ff. ; V. Moser 1929, 87.

25 Aber: $u$, z. B. petrubnif (fol. 685) Auch hier hat sich der Monophtong in der Schrift durchgesetzt, vgl. oben Anm. 22.

26 Siehe oben Anm. 22.

27 Vgl. Kranzmeyer 1956, 48; H. Paul 1989, 170; V. Moser 1929, $168 \mathrm{ff}$.

28 Vielfach auch mit ei. Eine Distribution der beiden Diphtonge im Hinblick auf betonte bzw. unbetonte Position war nicht feststellbar, vgl. V. Moser 1929, 171.

29 Entgegen der herrschenden Regel hier ai-Schreibung im nicht volkstümlichen Kirchenwort (vgl. V. Moser 1929, 171), wo sonst $e i$ und dessen graphische Varianten auftreten. Obgleich zur selben Wortkategorie gehörend, wird hail im Bair. regelmäBig mit ai bzw. seinen Entsprechungen geschrieben (V. Moser ebd.), auch hier, z. B. fol. 33r.

30 Siehe oben Anm. 29.

31 Siehe oben Anm. 28. 
Hand 3 - graphische Varianten: ai, ay, in ain, Gailteß, hailigen, ${ }^{32}$ tailen, payd (fol. $\left.80^{v}\right)$.

2.1.1.6 Vereinzelte $o$-Schreibung für mhd. $a / \hat{a}^{33}$

Hand 1 - z. B. antlozz (fol. 10 ), Doniel (fol. $44^{\mathrm{r}}$ ). ${ }^{34}$

Hand 2 - z. B. gemont (fol. 69'). ${ }^{35}$

Hand 3 - holbs (fol. 80v).

\subsubsection{7 $a$-Schreibung für mhd. $o$ vor $r$ oder Nasal ${ }^{36}$}

Hand $1^{37}$ - z. B. margens (fol. $17^{\mathrm{v}}$ ), warden (fol. 14 $4^{\mathrm{r}}$ ), wart (fol. 20 ${ }^{\mathrm{r}}$ ); wane $t$ (fol. $\left.21^{v}\right) .38$

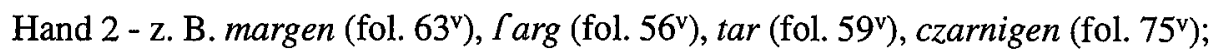
wanlt (fol. 51') ${ }^{39}$

Hand 3 - kein Beispiel.

2.1.1.8 $a$-Schreibung für mhd. $\hat{o}^{40}$

Hand 1 - kein Beleg.

Hand $2^{41}$ - z. B. kran (fol. $53^{\mathrm{v}}$ ), nat (fol. $57^{\mathrm{v}}$ ), tat (fol. $\left.55^{\mathrm{r}}\right) .^{42}$

Hand 3 - kein Beispiel.

2.1.1.9 Vereinzelte Delabialisierung von mhd. öu/iu, $>e i, e^{43}$

Hand 1 - z. B. erfrey (fol. $48^{v}$ ), kreitz (fol. 38v); getref ten (fol. 19v).44

32 Siehe oben Anm. 29.

33 Der vor allem im Mittel- und Südbair. (vgl. FrnhdG 1993, 45) erfolgte Übergang von mhd. kurzem und langem $a+$ offenem kurzem bzw. langem $o$ wird im Äfrnhd in "sorgfältigen" Hss. meist nicht gekennzeichnet (vgl. V. Moser 1929, 116 u. 143).

34 Aber: antlas tag (fol. $25^{\mathrm{r}}$ ), daniell (fol. $22^{\mathrm{v}}$ ).

35 Aber: gemant (fol. 70').

36 Durch den im Mittel- und Südbair. (H. Paul 1989, 171) sehr früh eingetretenen Wandel von $o$ vor $r$ und Nasalen + offenem $o$ kommt es zum Zusammenfall mit mhd. $a$ (s. oben Anm. 33), daher auch die Verwendung der Graphie $a$ für offenes $o$; jedoch kamen "die mundartlichen Wandlungen hier nur sehr beschränkt und fast nur im Äfrnhd. zum Ausdruck"; "im zweiten Viertel des 16. Jhs. stirbt dieses $a^{\prime \prime}$ (V. Moser 1929, 133).

37 Hier seltener als bei Hand 2, wo der Wandel vor $r$ nahezu regelmäßig durchgeführt wurde.

38 Aber: morgens (fol. $29^{v}$ ), worden (fol. 20v), wort (fol. 15); wonen (fol. $34^{v}$ ).

39 Aber: czornnikleicher (fol. $58^{\mathrm{r}}$ ).

40 Das mhd. $\hat{o}$ ist "in der Schriftsprache gewöhnlich erhalten geblieben". Die dialektalen Veränderungen werden nur in äfrnhd. Texten stärker reflektiert, doch begegnet im Bair. vereinzelt noch in der ersten Hälfte des 16 . Jhs. die Schreibung $a$ für mhd. $\hat{o}$. Im Großteil des Bair. beruht sie auf dem Zusammenfall von mhd. $\hat{a}$ und $\hat{o}$ im $o$-Laut mit offener Qualität. V. Moser 1929, 166f. und FrnhdG 1993, 38 u. 49.

41 Ziemlich häufig; nicht nur vor Nasal, sondern auch vor anderen Konsonanten.

42 Aber: kron (fol. 64 $4^{\mathrm{v}}$ ), not (fol. 73r), tot (fol. 70 r).

43 Vgl. FrnhdG 1993, 75f.; V. Moser 1929, 102ff.; K. Weinhold 1867, 82f. 
Hand 2 und 3 - kein Beleg für Entrundung.

2.1.1.10 Kontraktion von mhd. -age- vor $t>$ bair. $a i^{45}$

Hand 1 - z. B. ge fä̈tt (fol. 19v, $23^{v}$ ), maid (fol. $7^{v}$ ), trait (fol. $\left.2^{v}\right) .{ }^{46}$

Hand 2 - z. B. vber Cait (fol. 59v).

Hand 3 - kein Beispiel.

\subsubsection{Zum Vokalismus der Nebentonsilben}

2.1.2.1 Volle Vokale neben abgeschwächtem $e^{47}$

Beispiele:

gi-statt $g e$ -

Hand 3 - gileich (fol. $80^{\mathrm{v}}$ ).

vor-48 statt ver-

Hand 2 - vor partter (fol. 64).

Superlativsuffix $-i t^{49}$

Hand 1 - z. B. mynnigkleichi $\mathrm{t}$ (fol. $8^{\mathrm{r}}$ ), wunderleichift (fol. $8^{\mathrm{v}}$ ).

Hand 2 - z. B. Iunngiften (fol. 53v).

Zur Endung -ew etc. siehe unten 3.1, 3.2, 3.3.

Zur Endung -und im Part. Präs., siehe unten 3.6.1.

2.1.2.2 Dehnung des $i$ mit anschließender Diphtongierung im Bair. ${ }^{50}$

Hand 1 - z. B. Schepheleich (fol. $1^{\mathrm{r}}$ ).

Hand 2 - z. B. guldein (fol. 59v), wutreich (fol. 54r).

\subsubsection{Apokope}

Auffallend häufig ist die in der Hs. vorkommende Apokopierung in den üblichen ${ }^{51}$ Positionen. Eine genaue quantitative Analyse wurde allerdings nicht durchgeführt.

\subsubsection{Synkope S2 $^{2}$}

Im Vergleich zu Hand 1 (und 3) ist die Synkope beim zweiten Schreiber besonders zahlreich vertreten, vor allem die Ekthlipsis als Folge des $e$-Ausfalls ${ }^{53}$ (z. B. rinn, fol. $72^{r}$ ) ist relativ häufig zu finden.

44 Aber: erfrewe (fol. 21 ${ }^{\mathrm{r}}$ ), krewtz (fol. $3^{\mathrm{r}}$ ).

45 Vgl. H. Paul 1989, 137f.; V. Moser 1929, $113 \mathrm{ff}$.

46 Aber: magt (fol. $10^{\mathrm{v}}, 45^{\mathrm{r}}$ ).

47 Die in der Hs. vereinzelt vorkommenden vollen Vokale sind durchaus nicht anachronistisch, sondern sind, wenn auch nur gelegentlich, in frnhd. Texten zu finden; vgl. H. Paul 1989, 87ff.; FrnhdG 1993, $78 \mathrm{f}$.

48 "[...] im Bair. halten sich (seltenes) vor [...] bis ins 16. Jh." FrnhdG 1993, 78.

49 Vgl. FrnhdG 1993, 78.

50 Vgl. H. Paul 1989, 86.

51 Vgl. FrnhdG 1993, 81. 


\subsection{Zum Konsonantismus}

\subsubsection{Westgerm $k$ und auslautendes $g>$ Gutturalaffrikate $^{54}$}

Hand 1 - graphische Varianten: $c h, k h, c k h, c h k, g k h, z$. B. chum (fol. $9^{r}$ ), khinde (fol. $6^{\mathrm{r}}$ ), panckh (fol. $7^{\mathrm{r}}$ ), dinchk (fol. 35 ${ }^{\mathrm{v}}$ ), ringkh (fol. 18v)..$^{55}$

Hand 2 - graphische Varianten: ch, chk, kch, z. B. chuntt (fol. 79), plichk (fol. 67r), kchindernn (fol. 53) ${ }^{56}$

Hand 3 - kein Beispiel.

\subsection{2 $p$ für $b$ in unmittelbarem und mittelbarem Anlaut ${ }^{57}$}

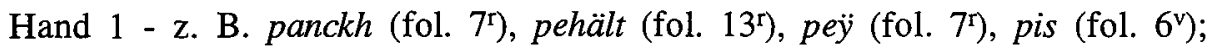
erparmert (fol. 1), gepet (fol. $\left.3^{\mathrm{v}}\right) .^{58}$

52 Vgl. FrnhdG 1993, 79f.

53 Vgl. spätbair. Schwund von -en nach Nasalen; H. Paul 1989, 170

54 Im älteren Frnhd. blieb die Gutturalaffrikate nur im Süd- und Mittelbair., hier bis an die Donau, sowie im östlichen Teil des Hochalem. erhalten, und zwar im Anlaut, im In- und Auslaut allgemein nur in der Verdopplung und nach $n$. Allerdings ist im Mittelbair. bei initialer Affrikate im Laufe des 15. Jhs. eine Rückbildung zum Verschlußlaut eingetreten.

In medialer und finaler Position nach den Liquiden $l, r$ wurde in den oben angeführten Gebieten die Affrikate schon früh zum Reibelaut vereinfacht, doch ist die Bezeichnung des Reibelauts im Äfrnhd. selten, so auch in der vorliegenden Hs.

Bezeichnung des Reibelauts durch die Graphie ch bzw. $h$ (vgl. V. Moser 1929, 60): Hand 1, z. B. werchen (fol. $3^{v}$ ), wurche (fol. $9^{v}$ ); Hand 2, z. B. karher (fol. $77^{v}$ ), karcher (fol. $77^{v}$ ), merch (fol. $51^{v}$ ), werchen (fol. $58^{r}$ ).

Nichtbezeichnung des Reibelauts: Hand 1, z. B. merckh (fol. $9^{v}$ ), werckh (fol. $1^{\mathrm{r}}$ ); Hand 2, z. B. markch (fol. $73^{\mathrm{v}}$ ), merkch (fol. $75^{\mathrm{r}}$ ).

Vgl. V. Moser 1951, 256ff. u. 1929, 59f. u. 64f.; FrnhdG 1993, 103.

Vor allem im Südbair. wurde auch das zu $k$ verhärtete auslautende $g$ zur Affrikate verschoben, vgl. O. Mausser 1933, 63.

55 Aber: kinde (fol. $21^{\mathrm{r}}$ ), tencken (fol. $43^{\mathrm{v}}$ ) mit Nichtbezeichnung der Gutturalaffrikate durch die Graphien $k$ und $c k$. Die Nichtbezeichnung der Affrikate durch $k$ ist im Vergleich zu den eindeutigen Graphien $c h, c k h$ usw. überhaupt vorherrschend. Vgl. V. Moser 1951, 257 u. 269.

Vereinzelt ist bei den Verhärtungsfällen von $g$ eine eindeutige Verschiebung zur Affrikate anzutreffen, doch überwiegt bei weitem die "vermittelnde" Graphie $g k$ bzw. einfaches $g, z$. B. ding $k$ (fol. 19v), anfang (fol. 18r). Vgl. Moser 1951, 275 u. FrnhdG 1993, 102.

56 Aber: kunt (fol. $75^{v}$ ). Auch hier ist $k$-Schreibung vorherrschend. - Das $g$ in Verhärtungsstellung wird hier hauptsächlich als $g$ oder $g k$ wiedergegeben, bisweilen tritt auch die Gutturalaffrikate in Erscheinung, z. B. Iunchfrav (fol. 54v). Vgl. oben Anm. 55.

57 Im Äfrnhd. ist initiales $p$ nicht nur ein Merkmal des Südbair, es ist wahrscheinlich auch noch im Mittelbair. erhalten (V. Moser 1951, 103ff.).

58 Im Anlaut ist die $p$-Schreibung zwar vorherrschend, aber es treten auch Abweichungen auf, die auch für das Süd- und Mittelbair. gelten: Einführung der $b$-Graphie in bestimmten Worten (brueder, fol. $2^{\mathrm{v}}$ ), Erweichung der Fortis in unbetonter Stellung (vornehmlich in der Vorsilbe be-, z. B. behuet, fol. $20^{\mathrm{r}}$; bey, fol. $2^{\mathrm{v}} ;$ Bis, fol. $6^{\mathrm{v}}$ ). Die Erweichung nach Sonorkonsonanten im indirekten Anlaut ist in der Hs. nur äußerst selten durchgedrungen, das $p$ ist vorherrschend (nur furbringe, fol. $32^{v}$; verbrin, fol. $48^{\mathrm{v}}$ ). Vgl. V. Moser $1951,105 \mathrm{f}$.

Das inlautende einfache zwischen Vokalen stehende $p$ in napel (fol. $50^{v}$ ) ist für das Bair., wo ein Übergang von $b+w$ erfolgt (siehe unten 2.2.3), nicht charakteristisch, es ist eher für das 


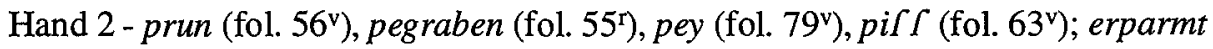
(fol. 51 $1^{\mathrm{r}}$, gepet (fol. $\left.75^{\mathrm{v}}\right) .{ }^{59}$

\section{Hand 3 - kein Beispiel.}

\subsection{3 $b$-Schreibung für $w$ und $w$-Schreibung für $b^{60}$}

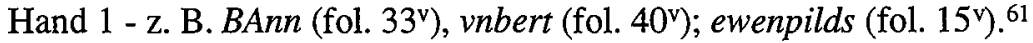

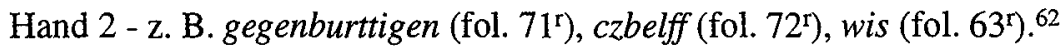

Hand 3 - kein Beispiel.

\subsubsection{Sonderfälle}

\subsubsection{1 $t / d$-Wechsel ${ }^{63}$}

Auffallend ist die vom zweiten Schreiber öfters verwendete Graphie $d$ für regelrechtes $t$, wie beispielsweise in auff $d u$ (3.Sg.Konj.Präs., fol. 52v), dacht (fol. 67r),

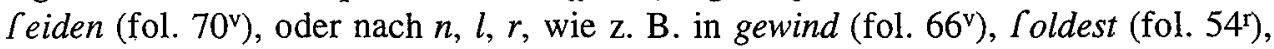
warden (fol. 59r). Daneben stehen auch "reguläre" graphische Varianten für die

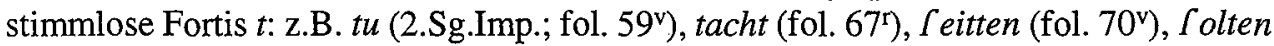
(fol. 54v), warten (fol. 59r).

Die $d$-Schreibung für $t$ nach $n, l, r$ findet sich auch beim ersten Schreiber, allerdings nur äußerst selten: z. B. hindenn (fol. $44^{v}$ ), wild (2.Sg.Ind.Präs. von wollen; fol. $48^{\mathrm{r}}$ ), virden (fol. $22^{\mathrm{r}}$ ).

\subsubsection{Sporadische $g$-Schreibung im Anlaut vor Vokal für $k$}

Das im Anlaut stehende etymologische $k$ wird $u$. a. vor Vokal auch im nördlichen und mittleren Ostobd. vereinzelt als $g$ wiedergegeben (FrnhdG 1993, 102), so auch bei Hand 2 beispielsweise in gattrein (fol. 54r), gattrey $(n)$ (fol. 66 $6^{\mathrm{r}}, 76^{\mathrm{v}}, 77^{\mathrm{r}, \mathrm{v}}, 78^{\mathrm{r}}$ ).

\subsubsection{Zur Assimilation von $m b>m m$}

Die Vereinfachung der Konsonantenverbindung $m b>m m$ tritt im Bair. mit Ausnahme des Südbair., wo sie seit 1300 belegt ist gewöhnlich nur zögernd ein (H. Paul 1989, 169; Kranzmeyer 1956, 106), auch in unserer Hs. ist $m b$ vorherrschend, bei

Hochalem.-Schwäb. bezeichnend; vgl. V. Moser 1951, 115.

59 Aber: brun (fol. 60v), betruben (fol. $74^{\mathrm{v}}$ ), bey (fol. 71 ${ }^{\mathrm{r}}$ ); vgl. unten Anm. 60. Die p-Schreibung ist bei Hand $2 \mathrm{im}$ allgemeinen vorherrschend, auch im Präfix pe-; $b$ statt $p$ in mittelbarem Anlaut ist hier nirgends vertreten. Vgl. oben Anm. 58 .

60 Häufig im Bair. seit dem Spätmhd. und im Äfrnhd., vgl. H. Paul 1989, 168; FrnhdG 1993, 85f. u. $104 \mathrm{ff}$.

61 Daneben: Wann (fol. 155), vnwert (fol. $40^{\mathrm{v}}$ ).

62 Daneben: gegenwurtige (fol. $71^{\mathrm{r}}$ ), bis (fol. $62^{\mathrm{v}}$ ).

63 Weinhold spricht von "scheinbarer Einheit von $t$ und $d "(1867,144)$. Diese ist erklärbar durch die Konsonantenschwächung und den Verlust des Stimmtons bei $d$, vgl. FrnhdG 1993, $162 f$.

Die $d$-Schreibung ist, obwohl ziemlich selten, seit der 2. Hälfte des 15 . Jhs. auch im Nord- und Mittelbair. anzutreffen, nicht im Südbair. Vgl. FrnhdG 1993, 91; V. Moser 1951, 158f. 
kattemer(en) (fol. 65 $5^{\mathrm{r}},<$ quatember, kotember) ${ }^{64}$ ist die Konsonantenfolge allerdings assimiliert und vereinfacht worden (H. Paul 1989, 169).

\subsubsection{4 $m$ zwischen zwei Liquiden $>$ bair. $w^{65}$}

In erbl (fol. $75^{\vee}$, Hand 2) mit der Schreibung $b$ für $w$, vgl. oben 2.2.3.

\subsubsection{5 $p$ - > b- in einigen Kirchenwörtern im Bair. ${ }^{66}$}

Hand 1 - brief ter (fol. 265). ${ }^{67}$

Hand 2 - brierter (fol. $67^{\mathrm{r}}$ ). ${ }^{68}$

\subsubsection{6 gx-Schreibung für chs}

Die nach V. Moser $(1951,281)$ erst im 16. Jh. belegte, vornehmlich bair. "Doppelschreibung" gx für die regelmäßige historische Graphie chs könnte einen Anhaltspunkt bieten für eine genauere Datierung von Hand 2, wo sie zwar nur fünfmal, aber konsequent für diesen Laut verwendet wird: wagx (fol. $56^{\mathrm{r}}, 65^{\mathrm{v}}$ hier zweimal), 66r), wagxs ${ }^{69}$ (fol. 67r). Der erste Schreiber gebraucht dagegen nur chs: wachs (fol. $\left.48^{\mathrm{r}, \mathrm{v}}\right)$.

\section{Morphologie}

\subsection{Zur Adjektivdeklination ${ }^{70}$}

Ausgesprochen bair. Merkmal sind im Äfrnhd. die sog. unechten ${ }^{71}$ Endungen -eu, die bis Ende des 15. Jhs. neben den zu -e abgeschwächten bzw. den auf Apokope beruhenden "Null"-Flexiven belegt sind. Unechte -ew finden sich im Sg./Pl.Akk.Fem. und im Pl.Nom./Akk.Mask.

Hand 1 - z. B. rechtew (fol. 1ㄴ, Sg.Akk.Fem.), heyligew (fol. 1x, Pl.Akk.Fem.).

Hand 2 und 3 - kein Beleg.

64 Vgl. M. Lexer 1986, 162. Das initiale ka- erklärt sich aus der Verdumpfung von qua- + bair. ko(vgl. H. Paul 1989, 141; siehe auch K. Weinhold 1867, 39) mit a-Schreibung für $o$ (vgl. oben 2.1.1.6).

65 V. Moser 1951, 95; vgl. P. Lessiak 1944, 85.

66 Vgl. H. Paul 1989, 168.

67 Aber: priefter (fol. $25^{\mathrm{v}}, 26^{\mathrm{v}}, 27^{\mathrm{r}}$ ).

68 Aber: prie $(\Gamma)$ ter (fol. $52^{\mathrm{r}}, 80^{\mathrm{r}}$ ).

69 Partitiver Genitiv (vgl. FrnhdG 1993, 332), deswegen auslautendes s.

70 Vgl. K. Weinhold 1867, 382ff.; FrnhdG 1993, $189 \mathrm{ff}$.

71 Allgemein obd. ist die reguläre Endung $-e u<$ mhd. $-i u$ im Sg.Nom.Fem. sowie im Sg.Instr.Neutr. und Pl.Nom./Akk.Neutr. 


\subsection{Zum Gebrauch des Demonstrativpronomens dew}

Die Form dew (< mhd. diu) tritt nur bei Hand 1 neben dem vorherrschenden die gelegentlich in Erscheinung, und zwar für den Sg.Nom. ${ }^{72} / \mathrm{Akk}^{73}$ Fem. und für den Pl.Nom. ${ }^{74} /$ Akk. ${ }^{75}$ Mask. und den Pl.Nom. ${ }^{76 / A k k .} .^{77}$ Neutr. Der zweite und der dritte Schreiber machen davon keinen Gebrauch. Diese Formen sind, so die FrnhdG (1993, 219), in Teilen des obd. Sprachraums noch bis zur Mitte des 15. Jhs. belegt.

\subsection{Zum Interrogativpronomen wew/weu}

Der bei Hand 1 und 2 vorkommende Instrumental ${ }^{78}$ bildet nach Weinhold (1867, 386) ein Unterscheidungsmerkmal zwischen dem Bairischen und dem Alemannischen, wo er "früh"79 verschwand. In Verbindung mit Präpositionen ist er zwar selten, aber "bis in das frühe 16. Jh. hinein" (FrnhdG 1993, 223) belegt.

\subsection{Zu den Genitiv- und Dativ-/Akkusativformen des Personalpronomens enkcher und enkch}

Die seit Ende des 13. Jhs. für den Plural gebrauchten Dualformen der 2. Person kommen überwiegend in bair. Texten vor und sind somit eine "dialektale Kennform des Bair." (FrnhdG 1993, 213; vgl. auch H. Paul 1989, 171 u. 221f.; K. Weinhold 1867, 367 u. 386). In der vorliegenden Hs. sind die Formen enkcher und enkch (fol. $76^{\mathrm{r}}$ ) nur beim zweiten Schreiber zu finden.

\subsection{Zum Gebrauch des Reflexivpronomens fich}

Interessant ist der allerdings nur einmal bei Hand 2 belegte Gebrauch des Reflexivpronomens $\int i c h^{80}$ anstelle von dich für die 2. Person Singular: trit (2.Sg.Imp.) als lang mit den rechten fus hinder Iich (fol. 57v). Diese unkorrekte Verwendung muß nicht unbedingt nur auf Schreibernachlässigkeit hindeuten, sondern kann auch sprachliche Interferenzen reflektieren, die auf den Einfluß des Slowenischen ${ }^{81}$ beruhen

72 Fol. $1^{\mathrm{v}}, 2^{\mathrm{v}}, 14^{\mathrm{r}}, 20^{\mathrm{r}}, 36^{\mathrm{v}}$.

73 Fol. $2^{\mathrm{r}}, 5^{\mathrm{r}}, 7^{\mathrm{r}}, \mathrm{v}, 14^{\mathrm{r}}, 22^{\mathrm{r}}, 35^{\mathrm{r}}$.

74 Fol. 22r; die Form kann aufgrund des nichtfestgelegten Genus von diet (vgl. M. Lexer 1986, 31) auch Fem. oder Neutr. darstellen.

75 Fol. $38^{\mathrm{r}}$

76 Fol. $6^{\mathrm{r}}, 35^{\mathrm{v}}$.

77 Fol. 39 r

78 Hand 1 - wew (fol. $28^{\mathrm{r}}, 31^{\mathrm{r}}, 32^{\mathrm{v}}$ ); Hand 2 - weu (fol. $51^{\mathrm{r}}$ ), wew (fol. $55^{\mathrm{v}}, 76^{\mathrm{v}}$ ).

79 Ein genauerer Zeitpunkt wird von Weinhold nicht angegeben.

80 Sonst regelhaftes dich, z. B. das du dich fcholft erneren des ewigen leben (fol. $75^{v}$ ); du dich williklleichen genaigt hart (fol. $78^{\mathrm{r}}$ ).

81 J. Stanonik (1957, 12 u. 41) betrachtet die Entstehung der Hs. im ehemaligen Krain als sehr 
könnten, wo das ungeschlechtliche Reflexivpronomen (<ide. *seue-; vgl. H. Krahe 1985,42 ) bekanntlich bei allen drei Personen und Numeri identisch ist. ${ }^{82}$

\subsection{Beobachtungen zum Verbum}

\subsubsection{Zur Endung des Partizip Präsens}

Besonders beliebt im Bair., wenn auch in alem. Texten vorkommend ( $\mathrm{K}$. Weinhold 1867, 337), ist das Flexiv -und(e) mit seinen orthographischen Varianten, das bis in das 17. Jh. belegt ist (FrnhdG 1993, 236; H. Paul 1989, 88). Im vorliegenden Gebethbuch ist es bei Hand 1 und 2 die vorherrschende Form, daneben tritt die zu -end abgeschwächte Endung weniger häufig in Erscheinung:

Hand 1 - z. B. an ligund (fol. $17^{v}$ ), geund (fol. 38 ), helffunt (fol. 47v), Sweÿgunde (fol. 28v); aber an rüeffend (fol. $7^{\mathrm{r}}$ ), [teend (fol. 34 ${ }^{\mathrm{r}}$ ).

Hand 2 - z. B. ligundt (fol. 53v), pitund (fol. 72v), ficzund (fol. 53v), ftevndt (fol. $53^{\mathrm{v}}$ ); aber nur ftend (fol. $72^{\mathrm{r}}$ ).

\subsubsection{Zu Formen von haben}

\subsubsection{Der Indikativ bzw. Konjunktiv Präteritum von haben}

Kennzeichnend für das Bair. sind die mit $e$ bzw. $i e^{83}$ gebildeten Präteritumformen von haben (H. Paul 1989, 277; FrnhdG 1993, 312). In der Hs. werden für das Präteritum durchgängig diese Formen gebraucht:

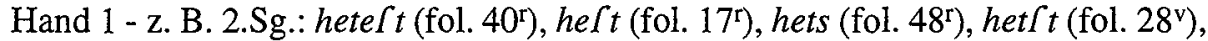

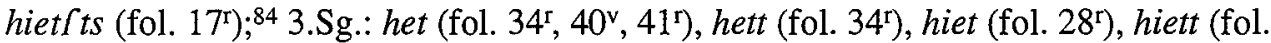
$25^{\mathrm{v}}$ ); 3.Pl.: hetten (fol. $17^{\mathrm{r}}$ ), hietten (fol. $19^{\mathrm{v}}$ ).

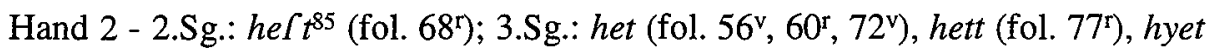
(fol. $60^{\mathrm{r}}, 66^{\mathrm{v}}, 68^{\mathrm{v}}, 71^{\mathrm{v}}$ ); 3. Pl.: hetten (fol. $64^{\mathrm{r}}, 68^{\mathrm{r}}, 72^{\mathrm{r}}$ ).

Hand 3 - kein Beispiel.

\subsubsection{Zum Gebrauch der kontrahierten Form han $^{86}$}

Die von Hand 1 und 3 gebrauchte kontrahierte Form han für die 1.Sg.Ind. (fol. $44^{\mathrm{r}}$ und $80^{v}$ ) wird hauptsächlich bis einschließlich erste Hälfte des 16. Jhs. gebraucht.

wahrscheinlich.

82 Analog vermutet auch Weinhold $(1867,369)$ slawischen Einfluß hinsichtlich der in der Oberpfalz verbreiteten Verwendung von sich statt uns zur Bezeichnung der reziproken Beziehung.

83 K. Weinhold (1867, 318f. u. 338) betrachtet nur die letzteren als bair.

84 Bei den vier letzten handelt es sich um kontrahierte Formen.

85 Vgl. oben Anm. 84.

86 Vgl. FrnhdG 1993, $311 \mathrm{f}$. 


\subsubsection{Zur 2.Sg.Imp. von $\int e^{8 n^{87}}$}

Im 15. Jh. existieren drei verschiedene Formen der 2.Sg.Imp. von Cein: wis, bis und $\int$ ey. Der Gebrauch der ältesten Form wis (<ide. * $\underline{\text { ues-) }}$ ) ist im wesentlichen auf den Norden des deutschsprachigen Raumes beschränkt. Dagegen ist bis, nach Auffassung Pauls eine Neubildung zur 2.Sg.Ind.Präs., seit dem 13. Jh. im Ober- und Mitteldeutschen allgemein verbreitet und bis in das 17 . Jh. nachweisbar. Der fruiheste Beleg von sei stamme, so W. Besch $\left(1967,310\right.$ ), erst aus dem Jahre $1477,{ }^{88}$ was einen nützlichen Anhaltspunkt für die Datierung von Hand 1 liefert. Diese wahrscheinlich im deutschen Südosten gebildete Neuerung kann sich neben bis relativ schnell behaupten, dennoch ist in obd. Texten des 15. Jhs., so auch im vorliegenden Gebethbuch, die ältere Form vorherrschend. In der Handschrift Ms 224 sind folgende Formen zu finden:

Hand 1, z. B. Bis (fol. $6^{\mathrm{v}}$ ), Pis (fol. $6^{\mathrm{v}}, 7 \mathrm{r},{ }^{\mathrm{v}}$ ), pi $\Gamma_{s}$ (fol. 24v); $e \ddot{y}\left(\right.$ fol. $6^{\mathrm{r}}, 27^{\mathrm{v}}$ ).

Hand 2, z. B. wis (fol. 63), wi $(\Gamma)$ (fol. $\left.64^{\mathrm{r}, \mathrm{v}}\right) ;{ }^{89}$ bis (fol. 61 ${ }^{\mathrm{v}}, 62^{\mathrm{v}}$ ), pis (fol. 69v,

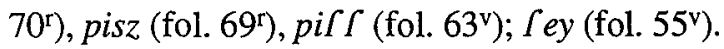

\subsubsection{Zur 3. Sg.Ind.Prät. von tun}

Die 3.Sg.Ind.Prät. von tun weist bei Hand 1 und 2 ein $e$ auf: tet (fol. $31^{\mathrm{v}}, 60^{r}$ ), dëtt (fol. 79r). Nach Angabe der FrnhdG $(1993,306)$ hält "der Sg. bis um 1500 'zäh am alten' $e$ fest". Nach K. Weinhold $(1867,303)$ ist es sogar noch im $16 \mathrm{Jh}$. vorherrschend.

\section{Zur Lexik}

\subsection{Das Suffix -nus und seine Varianten ${ }^{90}$}

Ausschließlicher Gebrauch der bair.-ostalem. Formen: ${ }^{91}$

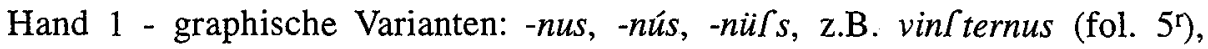
gedachtnús (fol. 50v), geleichnüfs (fol. $9^{\mathrm{r}}$ ).

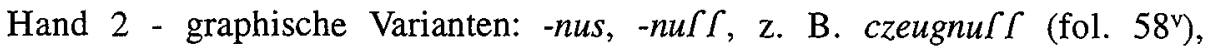
petrubnu $\int$ (fol. 68 ${ }^{\mathrm{r}}$ ).

87 Zu dem folgenden vgl. W. Besch 1967, 309f.; H. Paul 1989, 273f;; FrnhdG 1993, 309ff.

88 Laut FrnhdG $(1993,309)$ sei die neue Form dagegen erst seit dem 16. Jh. aufgekommen, was wir aber anhand der im vorliegenden Ms. auftretenden Formen widerlegen können, denn die Datierung von Hand 1 in das letzte Drittel des 15. Jhs. ist kaum zu bezweifeln; siehe unten 5 Untersuchungsergebnisse.

89 Die mit $w$ anlautenden Formen sind hier wohl eher ein graphischer Reflex, vgl. oben 2.2.3. Der Gebrauch von wis als norddeutscher Form könnte zwar auf fremde Vorlage hindeuten, ist aber weniger wahrscheinlich.

90 Mit Umlaut und den verschiedenen $s$-Graphien.

91 Vgl. W. Besch 1967, 225ff. 


\subsection{Einzelwörter}

Wörter, die für das Bair. charakteristisch sind, finden sich bei den ersten zwei Schreibern zum Teil neben "mundartneutralen" Entsprechungen:

Hand 1 - z. B. erichtags ${ }^{92}$ (fol. 49v), phintztag ${ }^{93}$ (fol. 49v); heint ${ }^{94}$ (fol. 36 ${ }^{v}$ ); Tenckhen $^{95}$ (fol. $40^{\mathrm{v}}$ ), ze wen $^{96}$ (fol. 16r).

Hand 2 - z. B. eritag (fol. 65 ), phincztag (fol. 58v); phaidt ${ }^{97}$ (fol. $75^{\mathrm{v}}$ ); dengken (fol. $67^{r}$ ), ze $\int e n^{98}$ (fol. $70^{r}$ ).

\section{Untersuchungsergebnisse}

\subsection{Hand 1}

Eine eindeutige Bestimmung der Schreibsprache im Hinblick auf Zeit und Raum ist für das 15. Jh. äußerst schwierig; die Handbücher machen oft nur vage und pauschale Angaben, die teils auf der Regellosigkeit - man denke nur an die Mannigfaltigkeit der graphischen Varianten -, teils auf der von der Forschung noch nicht vollständig erfaßten Fülle von schriftlichen Zeugnissen des ausgehenden Mittelalters oder auf noch nicht klar voneinander differenzierten Zuständen der Schreibsprachen mit fließenden Übergängen beruhen.

Vor allem das letztere erweist sich für das Ms NUKLj 224 als problematisch: das initiale $p$ für $b$ bildet in diesem Zeitraum nämlich noch kein klares Differenzierungsmerkmal zwischen dem Süd- und dem Mittelbair. ${ }^{99}$ Desgleichen stellt die Verwendung der Gutturalaffrikate im besagten Zeitraum noch kein Kriterium für eine deutliche Abgrenzung zwischen dem Süd- und dem Mittelbair. dar. ${ }^{100}$ Trotz Rückgang der Affrikate im Mittelbair. im Laufe des 15. Jhs. kann nicht einmal die Graphie $k$ als sicheres Indiz für den Verschlußlaut betrachtet werden, da die $k$-Schreibung sowohl im Mittel- als auch im Südbair. zur Wiedergabe beider Laute dient. ${ }^{101}$ Im Hinblick auf die anhand anderer Indizien ${ }^{102}$ durchgeführte Datierung von

92 Im Bair. für Dienstag, vgl. J. A. Schmeller 1872, 128 u. W. König 1981, 187; F. Tschirch 1983, 129.

93 Im Bair. für Donnerstag, vgl. oben Anm. 92.

94 Kontraktion aus hînacht mit der Bedeutung von dieser vergangene oder künftige Abend, vgl. J. A. Schmeller 1872, 1135.

95 J. A. Schmeller $1872,524$.

96 Aber: rechtten (fol. 16 ${ }^{\mathrm{T}}$ ).

97 J. A. Schmeller 1872, 444.

98 Aber: recht(en) (fol. $70^{\mathrm{r}, \mathrm{v}}$ ).

99 Siehe oben Anm. 57.

100 Siehe oben Anm. 54.

101 Siehe oben Anm. 55.

102 Siehe unten. 
Hand 1 um das Jahr 1472 ist südbairische Schreibsprache dennoch wahrscheinlicher, weil die Affrikate in Initialstellung relativ häufig durch charakteristische Graphien gekennzeichnet ist. ${ }^{103}$ Desgleichen ist die zum Teil gekennzeichnete Gutturalaffrikate bei Verhärtungsfällen von $g$ südbairisches Merkmal. ${ }^{104}$ Auch vom außersprachlichen Standpunkt ist Südbairisch dem Mittelbairischen vorzuziehen, denn nach Angabe des Besitzervermerkes auf dem vorderen Pergamentblatt der Hs. ${ }^{105}$ befand sich das Gebethbuch schon Mitte des 16. Jhs. in Ljubljana.

Die mit Hilfe des Wasserzeichens angesetzte Datierung um 1472 kann nach den im phonetisch-orthographischen bzw. morphologischen Bereich gewonnenen Erkenntnissen aufrechterhalten werden, aufschlußreich ist einerseits die Verwendung von ley für die 2.Sg.Imp. von sein, ${ }^{106}$ wodurch ein früherer Zeitpunkt, falls die Wasserzeichenbestimmung nicht korrekt sein sollte, kaum in Frage käme, andererseits spricht der Gebrauch des Demonstrativpronomens dew, das allgemein bis zur Mitte des 15. Jhs. im Obd. begegnet, ${ }^{107}$ gegen eine spätere Datierung.

\subsection{Hand 2}

Auch bei Hand 2 ist eine eindeutige Bestimmung der Schreibsprache aufgrund des oben dargelegten Sachverhaltes problematisch. Da aber auch hier die Gutturalaffrikate im Anlaut ziemlich häufig durch die Graphie ch deutlich gekennzeichnet ist, gelegentlich auch in den Verhärtungsfällen von $g$, und aufgrund der unten dargelegten Gründe eine noch spätere Datierung angesetzt ist, ist als Schreibsprache Südbairisch in Betracht zu ziehen, obwohl einige Besonderheiten auf Mittelbairisch hindeuten, wie das anlautende vorvokalische $g$ für $k,{ }^{108}$ der $d / t$-Wechsel ${ }^{109}$ und die "jüngere Diphtongierung", 110 die beide bei Hand 2 stärker ausgeprägt sind als bei Hand 1 .

Ein wichtiger Anhaltspunkt für die Datierung von Hand 2 ist zum einen die vom zweiten Schreiber verwendete Graphie $g x$ für chs, wofür sich nach V. Moser erst im 16. Jh. Belege finden, ${ }^{11}$ zum anderen bildet die zeitliche Grenze im 16. Th. u. a. der

Eine Abgrenzung zum Hochalem. bilden u. a. die nhd. Diphtongierung und die Entwicklung des alten Diphtongs $e i+a i$.

104 Siehe oben Anm. 55.

$105 \quad[. .$.

1550

zu dem $[. .$.$] daß er$

den fúe $\beta a b$ pran

hans fpynler holhper

zu labach.

Siehe oben 3.6.3.

Siehe oben 3.2 .

Siehe oben 2.2.4.2.

Siehe oben 2.2.4.1.

Siehe oben 2.1.1.3.

Siehe oben 2.2.4.6. 
Instrumental des Interrogativpronomens wew, der sporadisch nur noch zu Beginn des 16. Jhs. anzutreffen ist. ${ }^{112}$ Die "jüngere" Diphtongierung und die Schreibung $v$ als zweitem Bestandteil von Diphtongen sprechen $\mathrm{u}$. a. dagegen noch für das 15 . Jh. ${ }^{113}$ Folglich können wir, bei allem Vorbehalt gegen die oben angeführten Kriterien, als ungefähren Zeitraum für die Datierung von Hand 2 Ende 15./Anfang. 16. Jh. angeben. Untermauert wird die im Vergleich zu Hand 1 später angesetzte Datierung auch durch das Fehlen der "unechten" Endung -ew bei der Adjektivdeklilnation"114 und des Demonstrativpronomens dew. ${ }^{115}$

\subsection{Hand 3}

Bei Hand 3 kann aus Mangel an greifbaren sprachlichen Charakteristika eine nähere räumliche Eingrenzung der Schreibsprache im wesentlichen nicht vorgenommen werden. Vom Lautstand im Vokalismus und Konsonantismus läßt sich mit Sicherheit nur auf bairische Schreibsprache schließen, andererseits ist gegen eine Festlegung auf Südbairisch kein Einwand zu erheben.

Zur zeitlichen Bestimmung könnte die kontrahierte Form von haben herangezogen werden, die die 1. Hälfte des 16. Jhs. als oberste Grenze ${ }^{16}$ festlegt, doch kann der Text auch schon Ende des 15. Jhs. geschrieben worden sein.

\section{Literatur}

Ljubljana, National- und Universitätsbibliothek, Ms 224, Gebethbuch.

Besch, Werner: Sprachlandschaften und Sprachausgleich im 15. Jahrhundert: Studien zur Erforschung spätmittelhochdeutscher Schreibdialekte und zur Entstehung der neuhochdeutschen Schriftsprache. München 1967.

Briquet, Charles-Moïse: Les Filigranes. Bd. 1. Leipzig 1923.

Hartweg, Frédéric und Klaus-Peter Wegera: Frühneuhochdeutsch: Eine Einführung in die deutsche Sprache des Spätmittelalters und der frühen Neuzeit. Tübingen 1989.

Kos, Milko und F. Stelè: Srednjeveški rokopisi v Sloveniji. Ljubljana 1931.

Krahe, Hans: Indogermanische Sprachwissenschaft. Berlin; New York ${ }^{6} 1985$.

Kranzmeyer, Eberhard: Historische Lautgeographie des gesamtbairischen Dialektraumes. Wien 1956.

König, Werner: dtv-Atlas zur deutschen Sprache. München ${ }^{4} 1981$.

Lessiak, Primus und Eberhard Kranzmeyer: Die deutsche Mundart von Zarz in Oberkrain. Weimar 1944.

Lexer, Mathias: Mittelhochdeutsches Handwörterbuch. Bde. 1-3. Stuttgart 1974.

112 Siehe oben 3.3 .

113 Siehe oben Anm. 9.

114 Siehe oben 3.1.

115 Siehe oben 3.2.

$116 \mathrm{Vgl}$. oben 3.6.2.2. 
-----: Mittelhochdeutsches Wörterbuch. Stuttgart ${ }^{37} 1986$.

Mausser, Otto: Mittelhochdeutsche Grammatik. München 1933.

Moser, Virgil: Frühneuhochdeutsche Grammatik. Bde. I/1 und III/3. Heidelberg 1929, 1951.

Paul, Hermann: Mittelhochdeutsche Grammatik. Neu bearbeitet von Peter Wiehl und Siegfried Grosse. Tübingen. ${ }^{23} 1989$.

Piccard, Gerhard: Wasserzeichen Waage. Stuttgart 1978.

Reichmann, Oskar und Klaus-Peter Wegera (Hg.): Frühneuhochdeutsche Grammatik. Tübingen 1993.

Schmeller, J. Andreas: Bayrisches Wörterbuch. Bde. 1-2. München 1872, 1877.

Stanonik, Janez: Ostanki srednjeveškega nemškega slovstva na Kranjskem. Ljubljana 1957.

Tschirch, Fritz: Geschichte der deutschen Sprache. 2 Bde. Berlin ${ }^{2} 1983,{ }^{3} 1989$.

Weinhold, Karl: Bairische Grammatik. Berlin 1867.

\section{Povzetek \\ RAZISKAVA NEMŠKEGA PISNEGA JEZIKA V LJUBLJANSKEM POZNOSREDNJEVEŠKEM MOLITVENIKU}

Članek obravnava nemški pisni jezik v poznosrednjeveškem molitveniku NUKLj Ms 224. Jezikovna analiza je izvedena na fonetično-ortografskem, na morfološkem in na leksikalnem področju ob upoštevanju kriterijev, ki so relevantni za prostorsko in časovno razvrstitev. Besedilo so zapisali trije pisci. Zaradi določenih odstopanj pri zapisu besedila je jezik vsakega treh piscev ločeno prikazan. Cilj raziskave je na podlagi jezikovnih značilnosti natančneje določiti čas nastanka pričujočega manuskripta, ki je bil doslej le okvirno datiran v 15. stoletje. Datacija prve roke, katere besedilo je, poleg nekaterih srednjebavarskih značilnosti, zapisan v južnobavarskem pisnem jeziku, se ujema $z$ okvirno letnico 1472 , pridobljeno s pomočjo določitve vodnega znaka. Tudi druga roka kaže pretežno na južnobavarski pisni jezik, ki spričo nekaterih specifičnosti datira zapis v konec 15. oziroma začetek 16. stoletja. Zaradi pomanjkanja oprejemljivih jezikovnih značilnosti natančnejša določitev tretje roke znotraj bavarščine ni možna, le določitev zgornje časovne meje, ki je prva polovica 16. stoletja. 